\title{
The Design and Implementation of a Single, Double, and Triple Concurrent Beam In Situ Ion Irradiation TEM Facility
}

\author{
Daniel Bufford ${ }^{1}$ and Khalid Hattar ${ }^{1}$ \\ 1. Sandia National Laboratories, Radiation-Solid Interactions, Albuquerque, NM, USA
}

The study of irradiation-induced defects on the nanoscale is crucial for a fundamental understanding of displacement and implantation damage in various microstructures. Ion irradiation performed in situ inside of a TEM is a unique tool allowing direct observation of defect evolution on nanometer length scales in real time [1]. Most facilities use a single ion beam, but in many real radiation environments components are exposed simultaneously to a variety of particles with a spectrum of energies, while internal fission may create additional isotopes. Some studies have attempted to simulate the effects of multiple species by pre-implantation or sequential application of beams, however, several investigations [2-4] have revealed different defect behavior during sequential and concurrent application of the same beams. Knowledge of synergistic defect interactions during multiple beam irradiation is critical to understanding and predicting material behavior in complex radiation environments, like nuclear reactors, but cannot be reliably obtained with single beams.

Multiple beam in situ irradiation can provide this information, but its implementation poses substantially more challenges than a single beam facility. Separate ion sources, accelerators, and beam lines are typically required, as the physics involved in producing, accelerating, and steering ions are intrinsically tied to the charge state and mass of each ion species. Only a subset of in situ irradiation facilities currently employ multiple beams simultaneously [5], the newest of which is located at Sandia National Laboratories in the Ion Beam Laboratory. The in situ ion irradiation TEM (I ${ }^{3}$ TEM) facility consists of a JEOL 2100 TEM connected to both an EN Tandem (0.8-6 MV) and a Colutron G-1 accelerator (0.5-10 $\mathrm{kV}$ ). Energetic heavy ion beams from the Tandem induce large damage cascades, while lower energy beams from the Colutron may implant lighter ions within the TEM foil. Due to the differences in ion mass and energy, beams from both accelerators may be steered into the microscope through the same port, allowing irradiation by individual, sequential, or concurrent beams. Furthermore, the introduction of mixed source gases of identical ion beam rigidity (e.g. He and D2) into the Colutron permits irradiation by three species concurrently.

Here, $\mathrm{Au}$ films were deposited onto single crystal $\mathrm{NaCl}$ substrates, floated in distilled water and collected on Mo TEM grids, then annealed overnight at $300{ }^{\circ} \mathrm{C}$ to coarsen the grains. The samples were then exposed to combinations of single and concurrent beams consisting of $2.8 \mathrm{MeV} \mathrm{Au}^{4+}, 10 \mathrm{keV} \mathrm{He}^{+}$, and $10 \mathrm{keV} \mathrm{D}_{2}^{+}$. Still micrographs were collected before and after irradiation, and video was collected during. Cascades from individual $2.8 \mathrm{MeV} \mathrm{Au}^{4+}$ ions resulted in largely static defect clusters (Figure 1a), which moved only when a subsequent cascade occurred nearby. However, application of a concurrent $10 \mathrm{keV} \mathrm{He}{ }^{+}$beam caused 1-D oscillatory movement of some previously static dislocation loops (Figure 1b) [6]. During triple beam irradiation, defects from $2.8 \mathrm{MeV} \mathrm{Au}{ }^{4+}$ cascades caused repeated nucleation and annihilation of implanted cavities (Figure 1c,d). This work demonstrates the successful application of triple beam in situ ion irradiation, and the data reveal interesting defect behavior during multiple beam irradiation. These results suggest applicability of the technique to in situ studies of defect production in more complex irradiation conditions than can be achieved with single beams [7]. 


\section{References}

[1] JA Hinks, Nuclear Instruments \& Methods in Physics Research Section B-Beam Interactions with Materials and Atoms 267 (2009), p.3652-3662.

[2] JL Brimhall and EP Simonen, Journal of Nuclear Materials 68 (1977), p.235-243. [3]

C Chisholm, et al, Materials Transactions 55 (2014), in press.

[4] D Brimbal, et al, Acta Materialia 64 (2014), p.391-401.

[5] P Trocellier, et al, Nuclear Instruments \& Methods in Physics Research Section B-Beam Interactions with Materials and Atoms 266 (2008), p.3178-3181.

[6] K Arakawa, et al, Science 318 (2007), p.956-959.

[7] The authors thank D.L. Buller, J.A. Scott, M. Marshall, and B.L. Doyle for their assistance. This work was supported by the Division of Materials Science and Engineering, Office of Basic Energy Sciences, U.S. Department of Energy. Sandia National Laboratories is a multi-program laboratory managed and operated by Sandia Corporation, a wholly owned subsidiary of the Lockheed Martin Corporation, for the U.S. Department of Energy's National Nuclear Security Administration under contract DE-AC04-94AL85000.
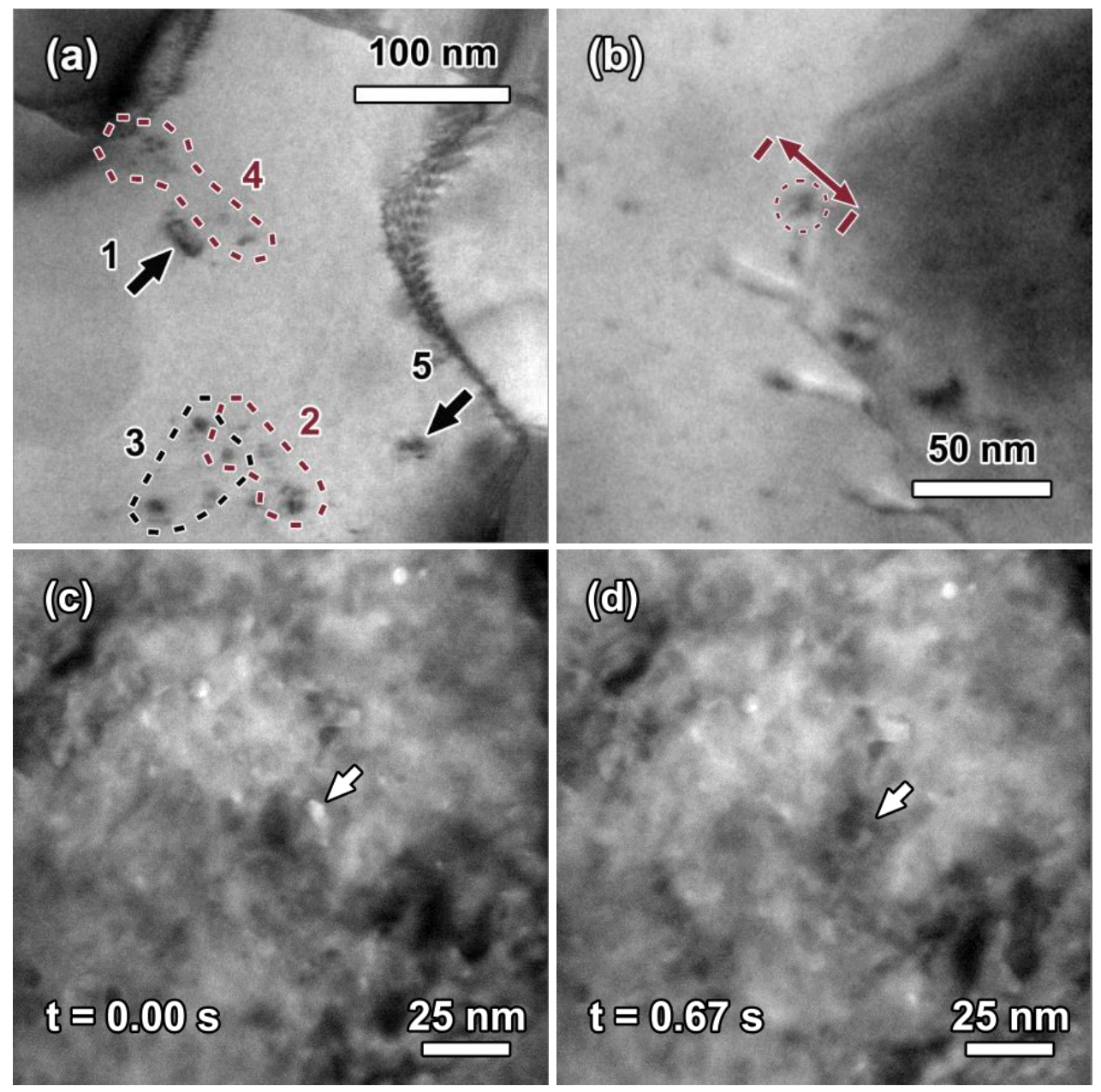

Figure 1. Transmission electron micrograph snapshots from video collected in situ during ion irradiation of Au with different beam combinations. (a) Static defect clusters of varying sizes remaining after 5 discrete $3.6 \mathrm{MeV} \mathrm{Au}{ }^{6+}$ collision cascades. (b) The extent of oscillatory movement of a dislocation loop created by an $\mathrm{Au}^{6+}$ collision cascade during concurrent $3.6 \mathrm{MeV} \mathrm{Au}{ }^{6+}$ and $10 \mathrm{keV} \mathrm{He}^{+}$ irradiation. (c,d) Disappearance of a cavity over $0.67 \mathrm{~s}$ during concurrent irradiation with $2.8 \mathrm{MeV} \mathrm{Au}{ }^{4}$, $10 \mathrm{keV} \mathrm{He}+$, and $10 \mathrm{keV} \mathrm{D}_{2}{ }^{+}$(at a fluence of approximately $1.1 \times 10^{16} \mathrm{He}^{+}-\mathrm{D}_{2}{ }^{+} / \mathrm{cm}^{2}$ and $3.9 \times 10^{13}$ $\left.\mathrm{Au}^{4+} / \mathrm{cm}^{2}\right)$. 